\title{
Development of the global simulation model of the heliosphere
}

\author{
Satomi Kamei ${ }^{1,4}$, Aoi Nakamizo ${ }^{2,4}$, Takashi Tanaka ${ }^{2,4}$, Takahiro Obara ${ }^{3,4}$, and Hironori Shimazu ${ }^{3,4}$ \\ ${ }^{1}$ Department of Earth and Planetary Sciences, Graduate School of Sciences, Kyushu University, 6-10-1 Higashi-ku, Fukuoka 812-8581, Japan \\ ${ }^{2}$ Department of Earth and Planetary Sciences, Faculty of Sciences, Kyushu University, 6-10-1 Higashi-ku, Fukuoka 812-8581, Japan \\ ${ }^{3}$ National Institute of Information and Communications Technology, 4-2-1 Koganei-shi, Tokyo 184-8795, Japan \\ ${ }^{4}$ CREST, Japan Science and Technology Agency, Kawaguchi-shi, Saitama 332-0012, Japan
}

(Received September 28, 2007; Accepted February 7, 2008; Online published May 29, 2009)

\begin{abstract}
The heliospheric structure ranging from the solar surface to the earth's orbit is self-consistently reproduced from a time-stationary three-dimensional (3D) magnetohydrodynamic (MHD) simulation. The simulation model incorporates gravity, Coriolis, and centrifugal forces into the momentum equation, and coronal heating and fieldaligned thermal conduction into the energy equation. The heating term in the present model has its peak at 2.8 solar radius $\left(R_{\mathrm{S}}\right)$ and exponentially falls to zero at greater distance from the solar surface. The absolute value of heating depends on the topology of the solar magnetic field so as to be in inverse proportion with the magnetic expansion factor. The results of the simulation simultaneously reproduce the plasma-exit structure on the solar surface, the high-temperature region in the corona, the open- and closed-magnetic-field structures in the corona, the fast and slow streams of the solar wind, and the sector structure in the heliosphere.
\end{abstract}

Key words: Solar wind, MHD simulation, CIR.

\section{Introduction}

There is a long history associated with the development of sun-earth-system models that try to predict the changes in the terrestrial environment caused by the disturbances on the solar surface following propagation through the interplanetary space (Dryer, 1998). Many early studies applied the physics-based model. Among these, the pioneering Hakamada-Akasofu-Fry kinematic model (Hakamada and Akasofu, 1982) is still currently in use by the U.S. space weather system. Since the large-scale structure and dynamics of the solar corona is dominated by the magnetic field, the source surface current sheet model was developed as an observed magnetic field data-driven model for the corona. This model can predict not only the sector structure but also the solar wind speed from the expansion factor (Wang and Sheeley, 1990). By extending the results up to the earth's orbit, model outputs can be compared directly with satellite observations.

In recent years, increases in computing speed have enabled the development of first-principle-based models. The hybrid model developed by Detman et al. (2006) combined the source surface current sheet model for the corona with the MHD solar wind model to predict the MHD parameters at the earth's orbit. Full MHD models from the solar surface to the solar wind region have also been developed by several authors (Linker et al., 1999; Riley et al., 2001; Manchester et al., 2004; Tóth et al., 2005; Shen et al., 2007). Although there are differences in the details for each model, these models can extrapolate the surface magnetic field together with self-consistently described plasma into the interplane-

Copyright (c) The Society of Geomagnetism and Earth, Planetary and Space Sciences (SGEPSS); The Seismological Society of Japan; The Volcanological Society of Japan; The Geodetic Society of Japan; The Japanese Society for Planetary Sciences; TERRAPUB. tary space.

In this paper, we report an attempt to develop an improved MHD simulation that unitedly reproduces quasistationary heliosphere from the solar surface to the earth's orbit from the observed solar surface magnetic field. When we try to reproduce the heliosphere extending form the solar surface to the earth's orbit, we must calculate both the fine structure around the corona and a global structure from the sun to near the earth's orbit simultaneously. In the following part of this paper, we first show how these difficulties are solved in our simulation model, and then we present some calculation results for a typical solar rotation period.

\section{Simulation Model \\ 2.1 Basic equation}

The basic equations of the simulations are the modified MHD equations written by dividing magnetic field $\mathbf{B}$ as $\mathbf{B}=\mathbf{B}_{0}+\mathbf{B}_{1}$ with $\mathbf{B}_{0}$ a fixed potential field and $\mathbf{B}_{1}$ deviation from $\mathbf{B}_{0}$ (Tanaka, 1994).

$$
\begin{gathered}
\frac{\partial \rho}{\partial t}+\nabla \cdot(\rho \mathbf{v})=0 \\
\frac{\partial(\rho \mathbf{v})}{\partial t}+\nabla \cdot\left(\rho \mathbf{v} \mathbf{v}+P \mathbf{I}+\frac{B^{2}-B_{0}^{2}}{2 \mu_{0}} \mathbf{I}-\frac{\mathbf{B B}-\mathbf{B}_{0} \mathbf{B}_{0}}{\mu_{0}}\right) \\
=\rho \mathbf{g}-2 \rho \Omega \times \mathbf{r}-\rho \Omega \times(\Omega \times \mathbf{r}) \\
\frac{\partial \mathbf{B}_{1}}{\partial t}+\nabla \times(\mathbf{v} \times \mathbf{B})=0 \\
\frac{\partial U_{1}}{\partial t}+\nabla \cdot\left[\mathbf{v} \cdot\left(U_{1}+P+\frac{B_{1}^{2}}{2 \mu_{0}}\right)-\frac{\mathbf{B}_{1}\left(\mathbf{v} \cdot \mathbf{B}_{1}\right)}{\mu_{0}}-\frac{\mathbf{B}_{0}\left(\mathbf{v} \cdot \mathbf{B}_{1}\right)}{\mu_{0}}\right. \\
\left.+\frac{\mathbf{v}\left(\mathbf{B}_{1} \cdot \mathbf{B}_{0}\right)}{\mu_{0}}\right]=Q-\rho \mathbf{v} \cdot \Omega \times(\Omega \times \mathbf{r})+\rho \mathbf{v} \cdot \mathbf{g}
\end{gathered}
$$


Table 1. Boundary conditions of the MHD and Poisson equations.

\begin{tabular}{c|c|c}
\hline Distance form the Sun & $1 R_{\mathrm{S}}$ & $200 R_{\mathrm{S}}$ \\
\hline Density & Fix & $\partial / \partial r=0$ \\
Velocity (parallel) & $\partial / \partial r=0$ & $\partial / \partial r=0$ \\
Velocity (perpendicular 1) & 0 & $\partial / \partial r=0$ \\
Velocity (perpendicular 2) & 0 & $\partial / \partial r=0$ \\
$B_{1}$ (radial) & 0 & $\partial / \partial r=0$ \\
$B_{1}$ (tangential 1) & $\partial / \partial r=0$ & $\partial / \partial r=0$ \\
$B_{1}$ (tangential 2) & $\partial / \partial r=0$ & $\partial / \partial r=0$ \\
$U_{1}$ & Fix & Fix $(P \rightarrow 0)$ \\
$B_{0}$ (line-of-sight) & observation & free \\
$B_{0}$ (perpendicular 1) & free & free \\
$B_{0}$ (perpendicular 2) & free & free \\
\hline
\end{tabular}

$$
U_{1}=\frac{\rho v^{2}}{2}+\frac{P}{\gamma-1}+\frac{B_{1}^{2}}{2 \mu_{0}}
$$

where $\rho, \mathbf{v}, P, \mathbf{r}, \mu_{0}, \mathbf{g}$, and $\gamma$ are the density, velocity, plasma pressure, position vector, the vacuum magnetic permeability, acceleration of gravity, angular velocity of solar rotation and polytropic index set as 5/3, respectively. These equations are generally called as continuity equation (1), equation of motion (2), induction equation (3), and energy equation (4). The energy equation (4) written through the modified total energy of plasma $U_{1}$ (Tanaka, 1994) includes the heating term $Q$. The equations are written in the rotating system, and they include the Corioli's force as well as the centrifugal force and gravity force.

\subsection{Boundary condition}

The inner boundary of the heliosphere is set at coronal base, which is approximately assumed to be 1 solar Radii $\left(R_{\mathrm{S}}\right)$, and the outer boundary is set at the near earth's orbit at $200 R_{\mathrm{s}}$. On the inner boundary, the density and total energy of the plasma are fixed, and field perpendicular velocities are set to 0 . The field parallel velocity is obtained from the Neumann condition. For the magnetic field, we put the observation data, which were obtained from Wilcox Solar Observatory, to the line-of sight component of $\mathbf{B}_{0}$. For $\mathbf{B}_{1}$, the radial component is set to 0 . On the outer boundary, total energy of the plasma is set so as the pressure becomes a small value near 0 . Other variables are solved under the Neumann condition. The boundary conditions of the MHD equation used for the present simulation are summarized in Table 1.

\subsection{Coronal heating}

To obtain a realistic solar wind with $\gamma=5 / 3$, some additional energy input is required (Lionello et al., 2001; Sittler et al., 2002). For the purpose of accelerating the solar wind to a high enough speed, we included an additional heating term to the energy Eq. (4) as

$$
Q=\rho q_{0}(r-1.0) e^{-r / L}+\nabla\left(\xi T^{2.5} \frac{\nabla T \cdot \mathbf{B}}{B^{2}}\right) \cdot \mathbf{B},
$$

where $q_{0}, L$, and $T$ are amplitude of heating, decay distance of heating (set to $1.8 R_{\mathrm{s}}$ ), and temperature (Sittler et al., 2002). The first term of the right handed side shows the ad hoc heating, while the second term shows Spitzer's heat conduction term parallel to the magnetic field line. This heat conduction is applied only inside $10 R_{\mathrm{s}}$. From Eq. (6),

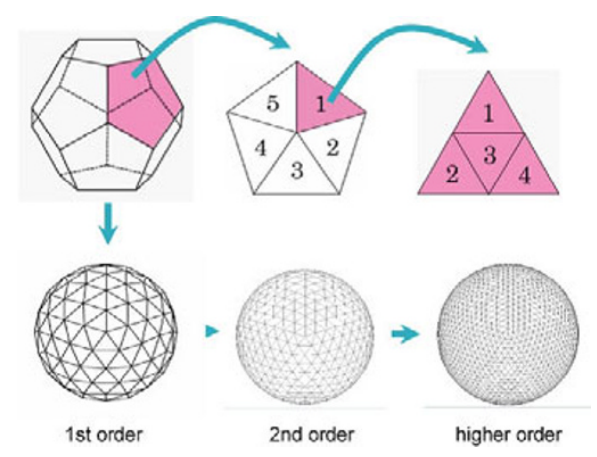

Fig. 1. Grid system made from a dodecahedron. One pentagon surface of regular dodecahedron is first divided into five right triangles, and then the generated triangles are divided into four smaller triangles.

it is clear that heating has its peak at $L+1=2.8 R_{\mathrm{s}}$ and subsequently decreases with a distance from the sun.

The magnetic field topology of the solar surface is important for solar wind speed. Therefore, we consider the magnetic field topology into the calculation of heating amplitude $q_{0}$. It is known that the flux tube expansion factor $f_{s}$, which is the ratio of the magnetic field intensity on the photosphere to that of respective points at extension along magnetic field, is inversely correlated with the velocity (Wang and Sheely, 1990). So, we set $q_{0}$ to be inversely proportional to $f_{s}$ written in the form

$$
f_{s} \equiv\left(\frac{r_{s}}{r_{s^{\prime}}}\right)^{2} \frac{B_{r_{s}}}{B_{r_{s^{\prime}}}}=\left(\frac{r_{s}}{r_{s^{\prime}}}\right) f_{s^{\prime}}
$$

where $r_{s}$ and $B_{r_{s}}$ are $1 R_{\mathrm{s}}$ and field intensity at the photosphere, and $r_{s^{\prime}}$ and $B_{r_{s^{\prime}}}$ denote distance from the solar center and field intensity at $r_{s^{\prime}}$. Given the magnetic field $\mathbf{B}$, the expansion factor $f_{s}$ is calculated by solving $\nabla \cdot\left(\mathbf{n} f_{s^{\prime}}\right)=0$ $\left(\nabla \cdot\left(-\mathbf{n} f_{s^{\prime}}\right)=0\right)$ with $\mathbf{n}=\mathbf{B} /|\mathbf{B}|$ along the away (toward) field lines, together with the boundary condition $f_{s}=1.0$ on the inner boundary. In addition to this inverse correlation considered through Eq. (7), $q_{0}$ is additionally reduced in the place where temperature is higher than 2.8 million $\mathrm{K}$.

\subsection{Grid system and numerical scheme}

When calculating a global system with a centrifugal configuration like the heliosphere, it is desirable to construct a spheroidal unstructured grid system having no apparent singularity. The grid system must also be tolerable for the calculation which treats both the fine structure around the center and wide-range structure extending the whole area simultaneously. To realize such a grid system, we first divide one pentagon surface of regular dodecahedron into five right triangles, adding a central point that inscribes a sphere. Next, we divide the generated triangles into four smaller triangles with all new points inscribing a sphere. Figure 1 shows this process of how we generate higher order splitting successively. By the fourth order splitting, 1922 grid points are generated. Stacking such spheres radially, a 3-D grid system is generated.

The control volume for the Finite Volume Method (FVM) calculation is hexagonal columns connecting the exocentric points of triangles on interfacing spheres. For each control volume, data for volume, area of interfacing surface, and normal and tangential vectors on the interfacing surface are 
prepared for the FVM calculation.

The numerical scheme used in the present simulation for the time integration of the conservative MHD equation is the FVM total variation diminishing (TVD) scheme with the monotonic upstream scheme for conservation laws (MUSCL) method, and the Van Leer's differentiable limiter originally developed by Tanaka (1994). For details, see Tanaka (1994).

\section{Numerical Results}

Here, we show the simulation results for 3-D structures of the solar corona and the solar wind for a typical solar rotation period. We have calculated the period of Carrington Rotation (CR) 2028 (2005, March 25-April 21) and then compared it with the observation is made at the earth's orbit using the solar wind data obtained by ACE (OMNI web service).

Figure 2 shows a 3-D close up view of the solar surface and solar corona as viewed from the earth on 2005 March 29. In this figure, the contour surface shows an isotemperature surface at 2.8 and inside of it is higher than 2.8. This temperature value is normalized by the surface temperature $0.8 \times 10^{6} \mathrm{~K}$. These high temperature regions correspond to the closed field regions, where the plasma is trapped. Red and blue lines show magnetic field lines. Here red (blue) lines show the away (toward) field lines. We can observe the origin of sector structure in the corona where toward and away sectors are separated by the high temperature region. Open field lines extending from one continuous region have a same color. The area of another-color open field lines is separated by the high-temperature region. These structures are a 3-D generalization of 2-D field, temperature and flow structures shown by Sittler et al. (2002).

Color shading on the solar surface shows plasma flux emitted from the solar surface. Yellow and red colors show the region from where solar wind plasma is supplied to the interplanetary space. Consequently, these regions can be looked upon as the coronal hole. Coronal holes are situated at the center of one continuous region having samecolor field lines. Compared with SOHO extreme ultraviolet image observed during CR 2028, positions of these coronal holes estimated from the flux exiting region almost coincide with dark regions seen in X-ray images.

From Fig. 2, we can see that the calculation has reproduced the solar and coronal fine structures near the sun. The coronal hole, sector structure, and high temperature corona are organized as a combined structure. As will be shown in Fig. 3, sector and high-speed flow structures in the interplanetary space are the extension of this organized structure near the sun.

Figure 3 shows the global solar wind structure on the ecliptic plane from the solar surface to the earth's orbit. White lines show the start longitudes of each quarter which were face to face with the earth on March 25, April 1, April 15, and April 22. The shading and contour lines in Fig. 3 show the magnetic sector and solar wind speed in the interplanetary space. Velocity is shown by the contour lines after the conversion from the rotating (calculating) frame to the non-rotating (observing) frame. In Fig. 3, the interplanetary magnetic structure exhibits four sectors.

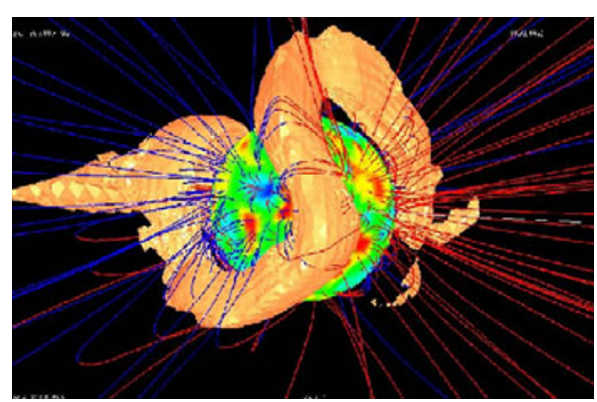

Fig. 2. A 3-D close up view of the solar surface and solar corona viewed from the earth on 2005 March 29. The contour surface shows an isotemperature surface at 2.8 . Temperature value is normalized by the surface temperature $0.8 \times 10^{6} \mathrm{~K}$. The coronal temperature outside the contour surface is about 1.7. Red lines show the away field lines and blue lines show the toward field lines. Color shading on the solar surface shows plasma flux emitted from the solar surface. The outward-flux area is indicated by warm colors.

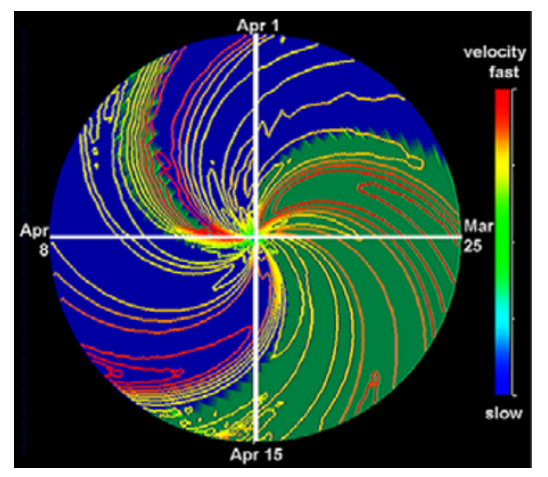

Fig. 3. The global solar wind structure in the ecliptic plane from the solar surface to near the earth's orbit. White lines show the start longitudes of each quarter which were face to face with the earth on March 25 (right), April 1 (top), April 8 (left), and April 15 (top). The shading and contour lines shows the magnetic sector (green-away, blue-toward) and solar wind speed (color bar: red-fast, blue-slow) in the interplanetary space.

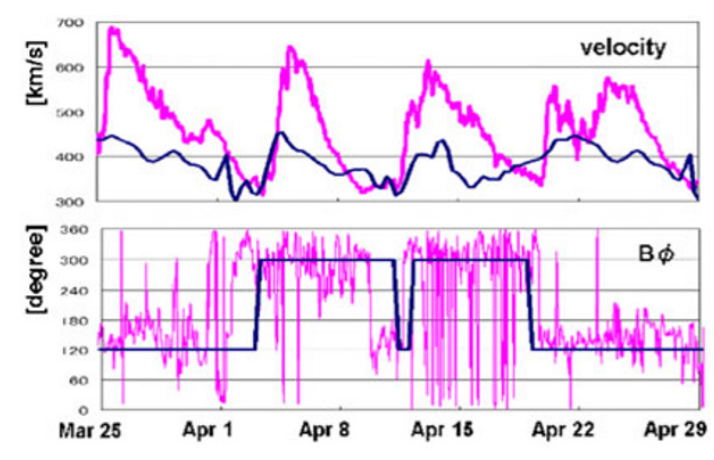

Fig. 4. Top pannel shows observed (pink) and calculated result (navy) of solar wind speed and bottom shows $B_{\phi}$ for ovserved data and $B_{r}>0$ (upper) or $B_{r}<0$ (lower) for calculation result. Observed near-Earth solar wind speed and magnetic field data by OMNI (http://omniweb.gsfc.nasa.gov). From the observed data during CR2028, it seems to have three speed peaks and 4 sectors including one very narrow sector around Apr 10.

This four sector structure reflects, in the ecliptic plane, the sectors shown in 3D in Fig. 2. The away sector seen on the right of Fig. 2 corresponds to the first away sector in the first quarter of Fig. 3 that starts on March 25 at the earth, 
as the beginning of CR 2028, and the toward sector seen on the left of Fig. 2 corresponds to the second toward sector in the second quarter of Fig. 3 that starts on April 1 at the earth. The third away sector seen in the third quarter at the earth is a very narrow away sector, and the fourth sector in the third and fourth quarter at the earth are both a toward sectors with a normal width.

Due to the solar rotation, the solar wind blows like a sprinkler seen in the non-rotating frame. In Fig. 3, three high-speed flows can be seen at the earth with their positions in the first away, second toward and fourth toward sectors. There is no high-speed flow in the third narrow away sector. In one sector, only one high-speed flow appears without consecutive high speed flows (Neugebauer $e t$ al., 2004). These flow and sector structures can be quite reasonably understood from the coronal structure shown in Fig. 2. The time recognized on the earth for the increasing of wind speed is shorter than that for decreasing. This characteristic resembles the observed feature of solar wind variation in the increasing and decreasing of speed (Gosling and Pizzo, 1999). In Fig. 3, no shock is generated associated with the CIR. Actually, there may not be an obvious CIR formation in the interval treated in this paper. However, we cannot draw a firm conclusion, because grid spacing in the present model is still too sparse for the shock resolution.

Figure 4 shows observed and calculated results for variations in solar wind speed and sector near the earth's orbit. Comparing the calculation results with these observation data, it is clear that the four sectors and three high speed winds seen in calculation are also observable . A narrow third away sector in the third quarter is similarly seen both in the calculation and in the observation data. As for the relative position of the sector boundary and fast flow, similar features are seen in Figs. 2, 3 and 4. At the earth, fast flow starts after the sector boundary has been passed, and fast flow does not restart until the passage of the next sector boundary. However, some discrepancies are seen. However, provided that the calculated and observed magnitude of the low-speed solar wind is the same, the magnitude of the calculated high-speed solar wind value is lower than the observed value. In other words, the contrast between highand low-speed flows is not sufficient in the calculated result. In addition, the decreasing speed of third fast flow is too rapid in the calculated than the observed data. These results suggest a further necessity for the improvement of the model. There are many uncertainties that prevent a modelobservation coincidence. There may still be many unknown factors in heating term, expansion factor, radiation energy balance, and magnetic field observation.

\section{Summary}

We have succeeded in constructing an MHD model to reproduce the heliospheric structure extending from the solar surface to the earth's orbit using a uniform triangular grid system with no apparent singular point. The calculation results have reproduced both of the fine structure around the corona and global structure from the solar surface to the earth's orbit. The tendency of a rapid increase and slow decrease of velocity seen on the earth and the relative position of the sector boundary, including the narrow sector, have been well reproduced in the model. Whereas three velocity peaks are seen at the earth's orbit in both the calculated and observed solar winds, the calculated results are still insufficient for the absolute speed of solar wind. This means that we need to improve this model further when using it for the real prediction of solar wind. For this purpose, we are going to continue variations and improvements of the model. In future research, we are going to adopt this model for the space weather prediction system operated in the National Institute of Information and Communication Technologies (NiCT), Japan.

Acknowledgments. Wilcox Solar Observatory data used in this study was obtained via the web site (http://quake. stanford.edu/ $\sim$ wso) courtesy of J. T. Hoeksema. The OMNI data were obtained from the GSFC/SPDF OMNIWeb interface at (http://omniweb.gsfc.nasa.gov).

\section{References}

Brio, M. and C. C. Wu, An upwind differencing scheme for the equations of ideal magnetohydrodynamics, J. Comput. Phys., 75, 400-422, 1988. Detman, T., Z. Smith, M. Dryer, C. D. Fry, C. N. Arge, and V. Pizzo, A hybrid heliospheric modeling system: Background solar wind, J. Geophys. Res., 111, A07102, doi:10.1029/2005JA011430, 2006.

Dryer, M., Multidimensional, magnetohydrodynamic simulation of solargenerated disturbances: space weather forcasting of geomagnetic storms, AIAA J., 36, 365-370, 1998.

Gosling, J. T. and V. J. Pizzo, Formation of corotating interaction regions and their three dimensional structure, Space Sci. Rev., 89, 21-52, 1999.

Hakamada, K. and S. Akasofu, Simulation of three-dimensional solar wind disturbances and resulting geomagnetic storms, Space Sci. Rev., 31, 3$70,1982$.

Linker, J. A., Z. Mikic, D. A. Biesecker, R. J. Forsyth, S. E. Gibson, A. J. Lazarus, A. Lecinski, P. Riley, A. Szabo, and B. J. Thompson, Magnetohydrodynamic modeling of the solar corona during Whole Sun Month, J. Geophys. Res., 104, 9809-9830, 1999.

Lionello, R., J. A. Linker, and Z. Mikic, Including the transition region in models of the large-scale solar corona, Astrophys. J., 546, 542-551, 2001.

Manchester, W. B., T. I. Gombosi, I. Roussev, D. D. De Zeeuw, I. V. Sokolov, K. G. Powell, G. Toth, and M. Opher, Three-dimensional MHD simulation of a flux rope driven CME, J. Geophys. Res., 109, A01102, doi:10.1029/2002JA009672, 2004.

Neugebauer, M., P. C. Liewer, B. E. Goldstein, X. Zhou, and J. T. Steinberg, Solar wind stream interaction regions without sector boundaries, J. Geophys. Res., 109, A10102, doi:10.1029/2004JA010456, 2004.

Riley, P., J. A. Linker, and Z. Mikic, An empirically driven global MHD model of the solar corona and inner heliosphere, J. Geophys. Res., 106, $15,889-15,901,2001$.

Shen, F., X. Feng, S. T. Wu, and C. Xiang, Three-dimensional MHD simulation of CMEs in three-dimensional background solar wind with the self-consistent structure on the source surface as input: Numerical simulation of the January 1997 Sun-Earth connection event, J. Geophys. Res., 112, A06109, doi:10.1029/2006JA012164, 2007.

Sittler, Jr., E. C., L. Ofman, S. Gibson, M. Guhathakurta, J. Davila, R. Skoug, A. Fludra, and T. Holzer, Development of multidimensional MHD model for the solar corona and solar wind, Solar wind 10, 2002.

Tanaka, T., Finite volume TVD scheme on an unstructured grid system for three-dimensional MHD simulation of inhomogeneous systems including strong background potential fields, J. Comput. Phys., 111, 381-389, 1994

Tóth, G., I. V. Sokolov, T. I. Gombosi, D. R. Chesney, C. R. Clauer, D. L. De Zeeuw, K. C. Hansen, K. J. Kane, W. Manchester, R. C. Oehmke, K. G. Powell, A. J. Ridley, I. I. Roussev, Q. F. Stout, O. Volberg, R. A. Wolf, S. Sazykin, A. Chan, B. Yu, and J. Kóta, Space Weather Modeling Framework: A new tool for the space science community, J. Geophys. Res., 110, A12226, doi:10.1029/2005JA011126, 2005.

Wang, Y. M. and N. R. Sheely Jr., The solar wind speed and coronal fluxtube expansion, Astrophys. J., 355, 726-732, 1990.

S. Kamei (e-mail: satomi@geo.kyushu-u.ac.jp), A. Nakamizo, T. Tanaka, T. Obara, and H. Shimazu 